\title{
Serious Games na saúde: aplicação de dispositivos non-touch em atividades de fisioterapia
}

\author{
Marcos Dias Vendramini ${ }^{1}$, Anderson Mine Fernandes ${ }^{1}$, Alexandre Rossi Paschoal ${ }^{1}$ \\ ${ }^{1}$ PPGI - Programa de Pós-Graduação em Informática \\ Universidade Tecnológica Federal do Paraná \\ Av. Alberto Carazzai, 1640 - 86.300-000 - Cornélio Procópio - PR - Brazil \\ marcosvendramini@hotmail.com, burnes@professorburnes.com, paschoal@utfpr.edu.br
}

\begin{abstract}
This article reports a Serious Games prototype for physiotherapy that use an Intelligence Specialist together with non-touch technologies. Our partial results shown the system viability. This system is not only for monitoring exercises but also to a tool to assist physical therapists in their jobs. The motivation is to apply this system as a complementary tool in physical therapy rehabilitation to help the patients and provide a quick feedback about exercises.
\end{abstract}

Resumo. Este artigo apresenta um protótipo de um Serious Games na área da fisioterapia implementado com Sistemas Especialistas usando tecnologias nontouch. Os resultados parciais demonstram a viabilidade do sistema. Este visa auxiliar o fisioterapeuta não apenas em monitorar os exercícios, mas em outras funcionalidades com games e reabilitação, de modo a fornecer aos pacientes $e$ profissionais um melhor feedback e motivação nos exercícios feitos.

\section{Introdução}

Em geral, na reabilitação fisioterápica aplica-se soluções como a dança e a equitação, o qual os jogos sérios podem ser uma ferramenta estratégica motivacional e adaptativa a dificuldade dos pacientes, independente de suas habilidades motoras e cognitivas [Guimarães and Nunes 2013]. Alinhado a isso tem-se tecnologias non-touch que vêm aumentando o potencial de suas aplicações em atividades terapêuticas e psico-motoras [Clua 2014], por meio a capturar os movimentos do corpo, fornecendo em tempo real o que está acontecendo [Barros et al. 2013]. Esta tecnologia tornam-se um fator motivacional e desafiador ao jogador/paciente na realização de suas atividades físicas.

Existem algumas soluções no mercado, sendo algumas pagas. Entretanto, são limitadas apenas ao dispositivo Kinect e voltado para corrigir os exercícios. Assim, este artigo descreve o resultado das pesquisas realizadas até o momento da implementação de um protótipo de serious game com sistemas especialistas via tecnologias non-touch para o aplicações em atividades físicas e de reabilitação. A seguir são apresentados alguns breves conceitos teóricos, trabalhos realcionados e a visão geral em detalhes do protótipo.

\section{Conceitos: tecnologias non-touch e jogos sérios}

Nos últimos anos houve uma grande evolução na maneira de interagir com o computador. Uma das grandes evoluções são os dispositivos non-touch (e.g. Kinect, Myo, LeapMotion e RealSense), que são tecnologias que reconhecem gestos, movimentos ou voz do usuário, sem a necessidade de contato direto com algum hardware, promovendo uma interação 
natural entre o usuário e o computador [Almeida 2013]. Segundo [Ghirotti 2009], as novas tecnologias non-touch são um passo para tornar interfaces naturais uma realidade no dia a dia do uso computacional.

Os Serius Games ou Jogos Sérios são aqueles em que além do fator diversão pode ser caracterizado também por outras finalidades (e.g. educação, exercícios físicos) [Tavares et al. 2014]. Normalmente, este tipo de jogo também permite ao jogador alcançar um determinado objetivo, aprender e se divertir [Michael and Chen 2006].

\section{Trabalhos Relacionados}

Após uma busca no Google Scholar e no IEEE obteve-se alguns artigos relacionados. Segundo [Balista 2013] o PhysioJoy utilizou o Kinect para três serious games que tratam de um tipo específico de lesão corporal ou cerebral. Os jogos trabalham com memorização, cognição, agilidade e membros no tratamento de lesões físicas e neurológicas.

O Physio-DHR [Rodrigues and Molz 2014] é voltado para a recuperação do equilíbrio corporal, por meio de mini-jogos para que pudessem treinar várias posições de equilíbrio.

Fernandes e Santos (2014) desenvolveram o Fisiogame, jogo com realidade aumentada/virtual para fazer exercícios de fisioterapia. Este reconhece o posicionamento do jogador mostrando na tela se está ou não sendo executada de forma correta [Fernandes et al. 2014]. Com o mesmo nome, mas aparentemente sem relação com anterior, o site http://fisiogames.com/ apresenta um jogo sério como serviço pago para a área de fisioterapia.

\section{Protótipo: visão geral e desenvolvimento do sistema proposto}

Serious Game tem se tornado uma tendência de uso na área da saúde para diversos tratamentos, muito pelo surgimento dos dispositivos que permitem a captura dos movimentos corporais. Assim, ficou mais fácil tornar seu uso em jogos terapêuticos e que tratam de reabilitação física [Clua 2014], o que até então era custoso e intrusivas, pois era necessário possuir diversos sensores pelo corpo do paciente [Hsieh 2012].

Diferente dos trabalhos relacionados, neste artigo não quer-se limitar ao tipo de exercício. O objetivo é apresentar um serious game para ajuda na fisioterapia, focar na reabilitação, dar um sistema de tempo real para o paciente e um sistema de relatório para profissional, bem como permitir a inclusão de novos exercícios não contidos no sistema, quando possível, pelo fisioterapeuta. Não se tem tal sistema hoje no mercado. Além disso, não criar um sistema apenas para Kinect, abrindo espaço para uso com outros dispostivos.

Entretanto, para este primeiro protótipo usou-se o framework do Kinect, que captura os pontos do corpo do jogador. Por exemplo, para o exercício de elevação lateral, os pontos a serem verificados são: pontos dos ombros, cotovelos e pulsos. Deve se calcular a distância entre esses pontos para poder estimar onde seria o ponto a ser alcançado pelo jogador. Para este cálculo, foi utilizado o cálculo da distância euclidiana, com o objetivo de validar e corrigir exercício físico. O sistema detectará o movimento dos braços do jogador e validará de acordo com as regras propostas pelo sistema especialista.

A Figura 1 esquematiza como funciona a captação da imagem do jogador através de um dispositivo non-touch de modo que o sistema especialista fará a validação dos 
movimentos e alertará e pontuará o jogador.

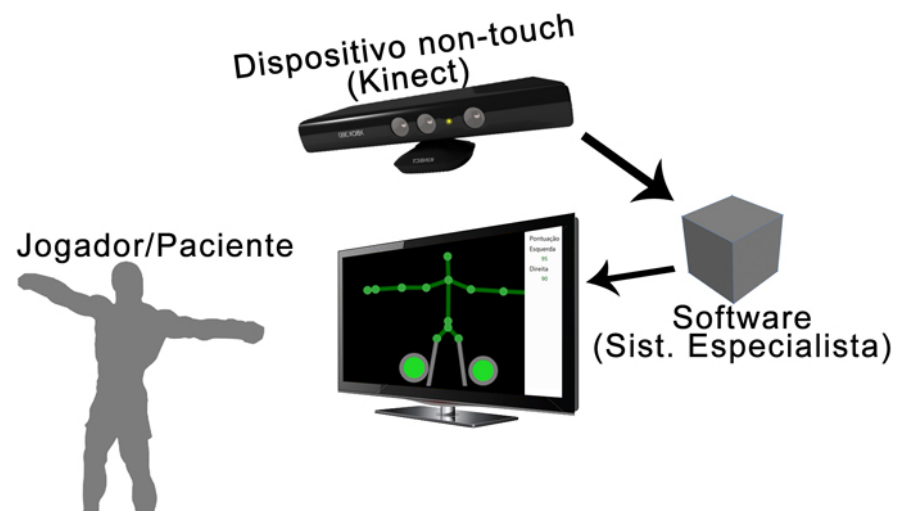

Figura 1. Aplicação da tecnologia non-touch no protótipo proposto

Os movimentos (Figura 2) do jogo irá consultar as regras propostas e avisar o jogador da corretude ou não. Por exemplo, o movimento com o braço torto, em referência ao ombro, cotovelo e pulso, emitirá um alerta visual vermelho (Figura $2 \mathrm{~A}$ ), enquanto que o braço reto, porém desalinhado referente ao corpo, emitirá um alerta visual amarelo (Figura 2 B). Já o braço na posição correta, mas sem alcançar o objetivo, o jogo emitirá um alerta visual verde (Figura $2 \mathrm{C}$ ), e para o braço na posição correta e tendo alcançado seu objetivo, o jogo mudará a posição do alvo a ser alcançado (Figura 2 D). Assim, a aplicação em um jogo envolve ganho de pontuação para o movimento correto ou perda de pontos para cada movimento errado. Cada jogo será personalizado para cada tipo de exercício.
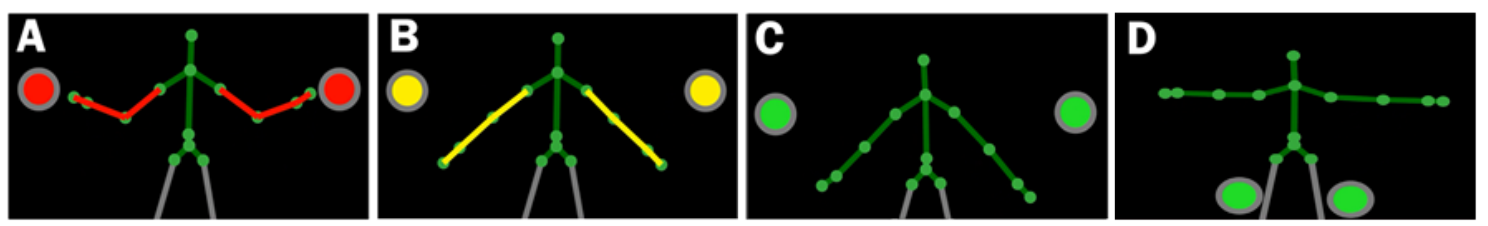

Figura 2. Tela inicial do Fisiogame: com a validação de cada movimento

\section{Conclusão e perspectivas futuras}

Os resultados esperados com a utilização deste jogo sério é que os pacientes fiquem mais motivados e satisfeitos durante as sessões dos tratamentos da fisioterapia, que se tenha uma maior aderência ao tratamento, assim reduzindo a chance de desistência. Segundo [Rossito 2013], uma das vantagens da utilização da reabilitação virtual é proporcionar uma maior motivação, graças as interfaces naturais interativas que incluem entretenimento a tarefa.

O protótipo já permite monitorar, alertar e acompanhar na corretude da execução de exercícios físicos e de reabilitação fisioterápica, tornando possível interpretar os movimentos em tempo real, de modo a auxiliar o fisioterapeuta. Espera-se melhorar a dinâmica/cenário do jogo, disponibilizar para outros dispositivos, não limitar a um conjunto de tipo de exercício, possibilitar que os exercícios que possam abranger às necessidades individuais de cada paciente, dar um sistema de tempo real para o paciente e um 
sistema de relatório para profissional, bem como permitir a inclusão de novos exercícios não contidos no sistema, quando possível, pelo fisioterapeuta.

\section{Referências}

Almeida, F. B.; Sales, A. B. (2013). Sistema interativo baseado em gestos para utilização de comandos no computador. Monografia de Conclusão de Curso de Graduação em Engenharia de Software - Universidade de Brasilia.

Balista, V. G. (2013). Sistema de realidade virtual para avaliação e reabilitação de déficit motor. SBGames. São Paulo, pages 16-20.

Barros, S., Passos, N., and Nunes, M. (2013). Estudo inicial sobre acidente vascular cerebral e serious games para aplicaçao no projeto. Revista GEINTEC-Gestao, Inovaçao e Tecnologias, 3(1):129-143.

Clua, E. W. G. (2014). Jogos sérios aplicados a saúde. Journal of Health Informatics, 6.

Fernandes, F. G., Santos, S. C., de Oliveira, L. C., Rodrigues, M. L., and Vita, S. S. B. V. (2014). Realidade virtual e aumentada aplicada em reabilitação fisioterapêutica utilizando o sensor kinect e dispositivos móveis.

Ghirotti, S. E.; Morimoto, C. H. (2009). Um sistema de interação baseado em gestos manuais tridimensionais para ambientes virtuais.

Guimarães, E. M. and Nunes, M. A. S. N. (2013). Prospecçao em jogos sérios para reabilitaçao de pacientes pós-avc. GEINTEC-Gestão, Inovação e Tecnologias, 3(2):147-156.

Hsieh, C; Chung, H. W. Y. C. L. (2012). Physical rehabilitation assistant system based on kinect. Proceedings of 2012 National Symposium on System Science and Engineering.

Michael, D. and Chen, S. (2006). Serious games: Games that educate, train, and inform. Thomson Course Technology - Boston, MA.

Rodrigues, E. and Molz, R. F. (2014). Physio-drh: desenvolvimento de game inclusivo para aplicação na recuperação do equilíbrio corporal. SBGAMES 2014.

Rossito, G. M. (2013). Estágio curricular i e ii manutenção e extensão do jogo "dance 2 rehab 3d". Trabalho de Conclusão de Curso de Ciência da Computação - Universidade do Estado de Santa Catarina.

Tavares, D., Silva, C., and Rousy, D. (2014). Um modelo de features para jogos sérios voltados à sa-úde: uma abordagem baseada em linha de produtos de software. WER14 - Workshop em Engenharia de Requisitos, Pucon, Chile. 